\title{
Disconnecting for the good: A network-oriented model for social contagion of opinions and social network interventions to increase adherence to social distancing
}

\author{
Eric Araújo $^{1}$, Mariza Ferro ${ }^{2}$, Gabrieli Silva ${ }^{2}$ \\ ${ }^{1}$ Departamento de Ciência da Computação (DCC/UFLA) \\ Caixa Postal 3037, 37200-900, Lavras - MG \\ ${ }^{2}$ Laboratório Nacional de Computação Científica (LNCC) \\ Av. Getulio Vargas, 333 - Quitandinha, Petrópolis - RJ, 25651-076 \\ ericeufla.br, mariza@lncc.br, gabrielielncc.br
}

\begin{abstract}
The pandemic of the new COVID-19 has raised many questions to a very connected society as to how to best respond to such a challenge at this current time. The best response so far is to call people for following the instructions from the World Health Organisation (WHO) as a way of reducing the spread of the virus and thus relieving the health system, striving to avoid a collapse. This work studies the spread of positive opinion on adhering to social distancing based on network topology and metrics using a network-oriented model for social contagion. It is shown that interventions based on social network measurements can be used to boost the spread of positive opinion about adhering to these measures. It is also shown that our model accounts for the relevance the health authorities have on encouraging people to partake in social distancing voluntarily.
\end{abstract}

Resumo. A recente pandemia do COVID-19 gerou questionamentos sobre como melhor responder à este desafio em uma sociedade conectada. A melhor resposta até o momento é convocar as pessoas para seguirem as recomendações da Organização Mundial de Saúde (OMS) de modo a reduzir o espalhamento do vírus e evitar um colapso do sistema de saúde. Este trabalho estuda o espalhamento de opiniões favoráveis à adesão ao distanciamento social baseado em métricas e topologias de redes usando um modelo baseado em redes de contágio social. É mostrado que intervenções baseadas em métricas de redes sociais podem ser usadas para aumentar o espalhamento de opiniões positivas sobre a adesão a essas medidas. Também é mostrado que o modelo considera a relevância que os orgãos de saúde possuem ao convencer a população de que o distanciamento social voluntário é importante.

\section{Introduction}

The World Health Organisation (WHO) declared COVID-19 a pandemic on March 11 2020 with over 100,000 cases of infected people by the coronavirus illness in over 100 countries. After this announcement, the whole world has been making efforts in order to contain the spread of the virus, or at least to flatten the curve of infected people as it would cause a collapse in the health systems, due to a lack of Intensive Care Units (ICUs) 
and ventilators for all of those who would develop severe and critical symptoms of the disease and as a consequence cause a larger number of deaths.

Many strategies have been discussed so far about what would be the best strategy to give time for hospitals and health centres to prepare for the worse case during the pandemic. Due to the lack of a vaccine, the instructions focus on increasing hygiene habits, as washing your hands more often with soap, the use of masks to cover mouth and nose in public spaces, staying home if possible, isolating people from the risk groups, restricting the gathering of people on the streets, concerts and big events, and encouraging people to adhere to social distancing. Some regions had also to adopt a hard lockdown, where people are obliged to stay home for a period, after losing control over the number of infected people. For example, the Italian region of the Lombardy region suffered thousands of deaths within weeks due to the lack of medical resources and prevention measures by the mayor of the city. In some countries, like Brazil, there is still a big concern with the outcomes of the pandemic in the economic spectrum. Other countries, like the United States, that were resistant to the idea of a hard lockdown now are also suffering with the increased number of patients in their hospitals.

To encourage people to stay home, artists and authorities have been broadcasting advertisements to make people aware of the importance of each individual action towards promoting social distancing and other cares needed to avoid the infection by the COVID-19. This effort is not equal in all countries, and even though encouraged by the WHO, still not taken seriously by all authorities. In these countries, the biggest motivation for the population to stay home or keep the social distancing comes from social media, TV channels and social spread of information. Besides the important task of motivating people to stay home, there is a battle to reduce the spread of the virus. Many models [Walker et al. 2020, Dandekar and Barbastathis 2020] have been developed in the past months in order to predict the numbers of people who are going to be infected. Other models try to predict when the peak of the infections are going to happen [Li et al. 2020, Zhou et al. 2020].

In this work, we look at the problem from a network perspective. The model proposed accounts for the motivation of people to adhere to social distancing based on homophily and social contagion. The Center for Disease Control and Prevention (CDC) defines social distancing (also called physical distancing) as "keeping space between yourself and other people outside of your home". This measure has proven to be one of the most effective ways of reducing chances of infection so far [Koo et al. 2020]. The aim of this work is to investigate how different configurations of people's networks could lead to different scenarios of social distancing adherence. Mainly, we look to answer the following questions:

1. Does the network topology affects the adherence to voluntarily social distancing in a pandemic scenario like COVID-19?

2. Are interventions based on social network traits efficient to increase the adherence to social distancing?

This work is organised as follow: Section 2 presents the related works. The methodology used to create the model and run the simulations is explained in Section 3 . The results obtained are presented in Section 4 and discussed in Section 5 . This latter section also presents the potential improvements in the model and other future works. 


\section{Related Works}

The coronavirus pandemic has called societies around the world for social distancing in order to avoid spreading the virus to other people, including closest family and friends. This is an altruistic and difficult decision that requires a firm decision to adhere or not to the preventive measures such as social distancing. [Webster et al. 2020] conducted a rapid review to list factors associated to quarantine during infectious diseases outbreaks. The researches have shown that adherence decisions are dependent on a few factors as (1) the knowledge people have about the disease and quarantine procedure, (2) social norms, (3) perceived benefits of quarantine and perceived risk of the disease, (4) practical issues such as running out of supplies or financial consequences of being out of work. [Webster et al. 2020] emphasises the importance of public health officials on providing on time, clear rationale for quarantine, as well as guaranteeing that supplies of food, medication and other essentials are provided. [Braunack-Mayer et al. 2013] evaluated the commitment of the Australian schools after the public health officials closed them as a strategy to mitigate the spread of the H1N1 virus in 2009. [Braunack-Mayer et al. 2013] conclude that the trust in public officials, government and the school as institution was the reason for the effective response to the strategy.

[Saunders-Hastings et al. 2017] proposed a mathematical model to predict the number of infected people, peak hospital care capacity, peak ICU capacity, deaths and the economic burden for future outbreaks of influenza in Ottawa, Canada. Authors used a social contact network structure for the transmission of the virus as three independent transmission-time/location steps throughout the day: household, school/work and community. In this scenario the vaccines and other treatments are considered for the agents in the model. [Chhatwal and He 2015] presented an Agent-Based Model (ABM) to evaluate the cost effectiveness of screening a percentage of the population during the epidemic of an infectious disease. They have shown the total cost of the health maintenance of advanced stages, treatment costs and screening costs in relation to the reduction of deaths and new infections due to screening.

[Centola 2018] presented a very good methodology for understanding how behaviour spreads in complex networks. His study is a very important foundation of what this work is going to evaluate, that is, what is the importance of network structure, homophily and social ties on the spread of COVID-19 and to the self-isolation adherence. [Araújo 2018] developed a model for the spread of healthy behaviour and social contagion considering people's traits and their ego networks to predict future states for their physical activity levels. [van Woudenberg et al. 2019] proposed a study to understand different strategies of interventions in order to increase the physical activity level of a group of children and teenagers at Dutch schools based on their networks. The model proposed in this research is network-oriented and time-dependent as the agents states affect the state of others over time [Treur 2020].

Recent modelling researches on COVID-19 are focused mainly on the epidemiological models, like SEIR (Susceptible, Exposed, Infectious, Recovered) in order to describe the epidemic and assess the impact of social isolation policies [Lin et al. 2020, Fang et al. 2020, Kucharski et al. 2020]. Some of them propose an agent-based model to simulate isolation interventions and understand the impact to reduce the spread of COVID-19. The work of [Klôh et al. 2020] uses an ABM combined with the SEIR model 
to simulate interventions and understand the impact to reduce the spread of COVID-19 considering the socioeconomic conditions of Brazil. The simulations have heterogeneous populations, numbers of Intensive Care Units and study different scenarios to show how the interventions can influence the spread of the virus.

[Wolfram 2020] proposed an ABM of COVID-19 that models the variability of interaction rates between agents and the structure of the in-person contact network. The author point out as findings that the structure of the contact network matters in the velocity of the spread and that reducing interaction between communities flattens the curve and reduces the average total infection rate. This work is similar to ours only in the sense of consider a social network to model the spread of COVID-19.

This is an initial effort to model the spread of COVID-19 and the adherence to social distancing as a health policy to avoid the spread of the virus. As far as our literature review could go this is the first work that tries to understand how social distancing adherence spreads using a network-oriented model approach in the context of a pandemic like COVID-19.

\section{Model and Simulations}

According to [Sokolowski and Banks 2011],

"Simulation is a good way to understand social processes because it can be used to predict the features of an environment, such as the changing demographics of a region over the course of time; or forecast changes such as business trends".

In this work we are interested on social processes of adherence to a new behaviour as a result of the COVID-19 pandemic in a network where the nodes are the people and the edges are the relationships.

The model presented in this work considers the following dynamics of social relations and the pandemic of the COVID-19:

1. People are more willing to partake in social distancing if their closer friends and family are following the guidelines;

2. People are more easily convinced that they should adhere the social distancing when they know someone who got the virus and developed severe or critical symptoms;

3. Relationships are built based on homophily, that is, people with closer characteristics tend to have stronger bounds;

4. Governments interventions in favour or against preventing the spread of the virus affect the whole population and, in that case, a risk mechanism is achieved in each agent considering relaxing the measures and the potential drawbacks of their decision.

\subsection{Social network structures and homophily}

The study of social networks encompasses the representation of social phenomena in social science contexts [Freeman 1989]. Therefore, social networks are representations in which relevant aspects of social phenomenon in a specific scenario are expressed in 
ways more fit to the scientific scope [Hennig et al. 2012]. The networks are made of nodes (or vertices) and edges (or links) as their main elements. For our model, the nodes represent people, while the edges represent the relationships, that is, who is connected to whom in real life. To account for the complexity of people and their social dynamics, the model is built upon the assumption that relationships are usually homophilic [McPherson et al. 2001]. That is, people with similar interests and characteristics tend to build closer relationships. This will affect the amount of time people spend together.

The difference between two persons traits is used to calculate their link strength as shown in Equation $11 . \omega_{i, j}$ is the link strength between two nodes $i$ and $j$. The network is bidirectional, that is, $\omega_{i, j}=\omega_{j, i}$. trait $_{i}^{k}$ is the $\mathrm{k}$-th trait for node $i$, where each node (or agent) has $n$ traits. All trait values are float numbers between 0 and 1 .

$$
\omega_{i, j}=\frac{\sum_{k=0}^{n-1} 1-\mid \text { trait }_{i}^{k}-\text { trait }_{j}^{k} \mid}{n}
$$

Each node represents a person with traits. The traits age, education level, socioeconomic situation and gender are used to define the homophily and connection strength between the nodes. age is normalised using linear scaling. Gender is 0 for male and 1 for female. The traits quarantine opinion $(q)$ and threshold for changes $(\tau)$ are used to define how the opinions about social distancing spread through the connections.

The network topologies used for this study are (1) Erdos-Renyi [Erdős and Rényi 1960], (2) Barabasi-Albert [Barabási and Albert 1999] and (3) Small-world [Watts and Strogatz 1998]. The different configurations proposed are important to account for simulations of different social contexts. It is expected, for instance, that favelas (Brazilian slums) present a denser network than wealthier neighbourhoods where people live in buildings.

\subsection{Opinion Contagion Model}

As explained in Section 2, one of the most important ways of mitigating the spread of infectious diseases is by promoting social distancing among the population. There are two ways people can adhere to this behaviour: (1) voluntarily and (2) obligatory. Case (1) is a result of a person who is convinced that she should stay without physical social contact as a way of caring for her and society's health. Case (2) is a result of an implemented policy by health authorities. In this case it is not acceptable for people to stay on the streets for the risk of a penalty (fine, arrest, etc). This model accounts for (1).

The voluntary social distancing is a result of an efficient strategy by the health authorities on communicating the risks of the disease and instructing on how to proceed. In these cases, it is expected that most of the citizens will have enough information to trust the preventive measures and adopt them. The voluntary social distancing can be also chosen by social contagion. That is, if most people someone knows take the measures seriously, the chances to adherence are higher then if most of the person's contacts are not following the protocol.

To simulate these situations, we consider that each agent has an opinion variable about how willing is the agent to adopt a social distancing behaviour (agent's opinion - $q$ ). This variable is 0 when the person is totally against the protocol, and 1 means total agree- 
ment with the need for it. Each agent has also a threshold (threshold for changes $-\tau$ ) which defines when agents will change their status as people who are following the social distancing protocol. Differential equations from the emotion contagion model presented by [Araújo and Treur 2016] combined with the spiral model presented by [Bosse et al. 2009] are used to describe the changes in agents opinions over time as shown below. The same approach has been used previously for behaviour contagion, emotion contagion and opinion contagion in social network contexts [Araújo 2018].

As described in [Araújo and Treur 2016], the overall contagion process is calculated in terms of the connection weight $\left(\omega_{i, j}\right)$ given by equation 1 . The impact caused by agent $j$ on agent $i$ is given by Equation 2, where $q_{j}$ is the opinion of agent $j$ about adhering to the protocol.

$$
\operatorname{impact}_{j, i}(t)=\omega_{i, j} q_{j}(t)
$$

The overall contagion strength $\omega_{i}$ to $q_{i}$ represents the total effect from all nodes connected to $i$. It is given by Equation 3 .

$$
\omega_{i}=\sum_{j \neq i} \omega_{i, j}
$$

The overall impact caused by all neighbours of agent $i$ is calculated as agg impact ${ }_{i}$, given in Equation 4 .

$$
\operatorname{agg} \operatorname{impact}_{i}(t)=\sum_{j \neq i} \frac{\omega_{i, j} q_{j}(t)}{\omega_{i}}
$$

The effect of the aggregated impact for changing the agents' opinions is given by the advanced logistic function defined by $q^{*}$ (Equation 5).

$$
q_{i}^{*}=\text { alogistic }_{\sigma, \tau}\left(\text { agg_impact }{ }_{i}\right)
$$

where alogistic $\sigma, \tau$ is given by Equation 6 ,

$$
\operatorname{alogistic}_{\sigma, \tau}(x)=\left[\frac{1}{1+e^{-\sigma\left(x-\tau_{i}\right)}}-\frac{1}{1+e^{\sigma \tau_{i}}}\right]\left(1+e^{-\sigma \tau_{i}}\right)
$$

where $\tau_{i}$ is the threshold for agent $i$. This will define from which point the influences from their peers start to affect their opinion. $\sigma$ defines the steepness of the slope of the advanced logistic function. In this work we tested values between 1 and 10 for $\sigma$.

The spirals model presented by [Bosse et al. 2009] have been used to account for the health authorities actions. $\beta$ relates to how strongly the health authorities are engaged in reinforcing the social distancing protocol to the population. This value varies from 0 to 10 , and is equal to all agents. The dynamic temporal changes in the agents' opinions is given by Equation 7 .

$$
\Delta q_{i}(t+\Delta t)=q_{i}(t)+\eta_{i}\left[\beta P I+(1-\beta) N I-q_{i}(t)\right] \Delta t
$$


The upward and downward direction of the changes in the agents' opinions depends on $\beta$, and the speed factor for the changes on parameter $\eta_{i}$. $\eta_{i}$ depends on the current opinion of the agent, and is defined by a beta distribution function with parameters $a$ and $b$ equal to 5. That means that the closest the agent's opinion is to one extreme, the slower the changes will occur.

Finally, $P I$ and $N I$ (Equation 8 ) are the positive and negative impact of received opinions from the agent's neighbours. If $\beta=1$ the receivers are only susceptible to positive influences, and if $\beta=0$ then the agent is only susceptible to negative influences. For this model we use the same $\beta$ to everyone assuming it represents the efforts of health authorities on promoting the protocol.

$$
\begin{aligned}
& P I=1-\left(1-\mathbf{q}_{i}^{*}\right)\left(1-q_{i}(t)\right) \\
& N I=\mathbf{q}_{i}^{*} \cdot q_{i}(t)
\end{aligned}
$$

At each time step, the model updates the agents' opinions and decides if they are going to adhere to social distancing or not. The chances are calculated based on their opinion levels.

\subsection{Network-based interventions}

Intervention is an action designed to bring about change in a target. This work investigated how interventions based on network traits will improve the overall social distancing adherence. The targeted agents in these simulations had their their social distancing opinion set to 1 with no possible changes throughout the simulation. For that, we tested 4 scenarios: (a) no interventions, (b) boost the opinion of $10 \%$ of agents with higher betweenness centrality, (c) boost the opinion of $10 \%$ of agents with higher closeness centrality and (d) boost the opinion of $10 \%$ of agents with higher eigenvector centrality [Hennig et al. 2012].

The interventions were performed in the 3 different network topologies (BarabasiAlbert, Erdos-Renyi and Small-world) and for 9 combinations of $\beta$ and $\sigma: \beta \times \sigma$ : where $\beta=\{0.1,0.5,0.9\}, \sigma=\{1,5,10\}$. In total there were 108 scenarios. Each simulation was ran 100 times, with a total of 10,800 runs for the entire process of opinion change. The simulations stopped when the opinions were not altered anymore.

\section{Results}

This section will present the results obtained by the simulations. As previously explained, there were 108 different scenarios considering no interventions and other network-based interventions in the network. The aim is to describe how altering $10 \%$ of the nodes would contribute to a higher adherence to social distancing habits in a group of connected people.

Firstly, it is important to understand the influence exerted by $\beta$ and $\sigma$ in the model. For that, the simulations were ran with no interventions for a different set of values for both variables:

- $\beta \in\{0.1,0.3,0.5,0.7,0.9\}$

- $\sigma \in\{1,5,10\}$ 
As it is shown in Figure 1, a higher $\sigma$ and $\beta$ values (Beta and Sigma axis) improve the overall opinion of all agents (Opinion axis) on adhering to the protocol. More specifically, for $\beta>0.5$ and $\sigma>5$ the overall opinion becomes positive towards the adoption of social distancing behaviour.
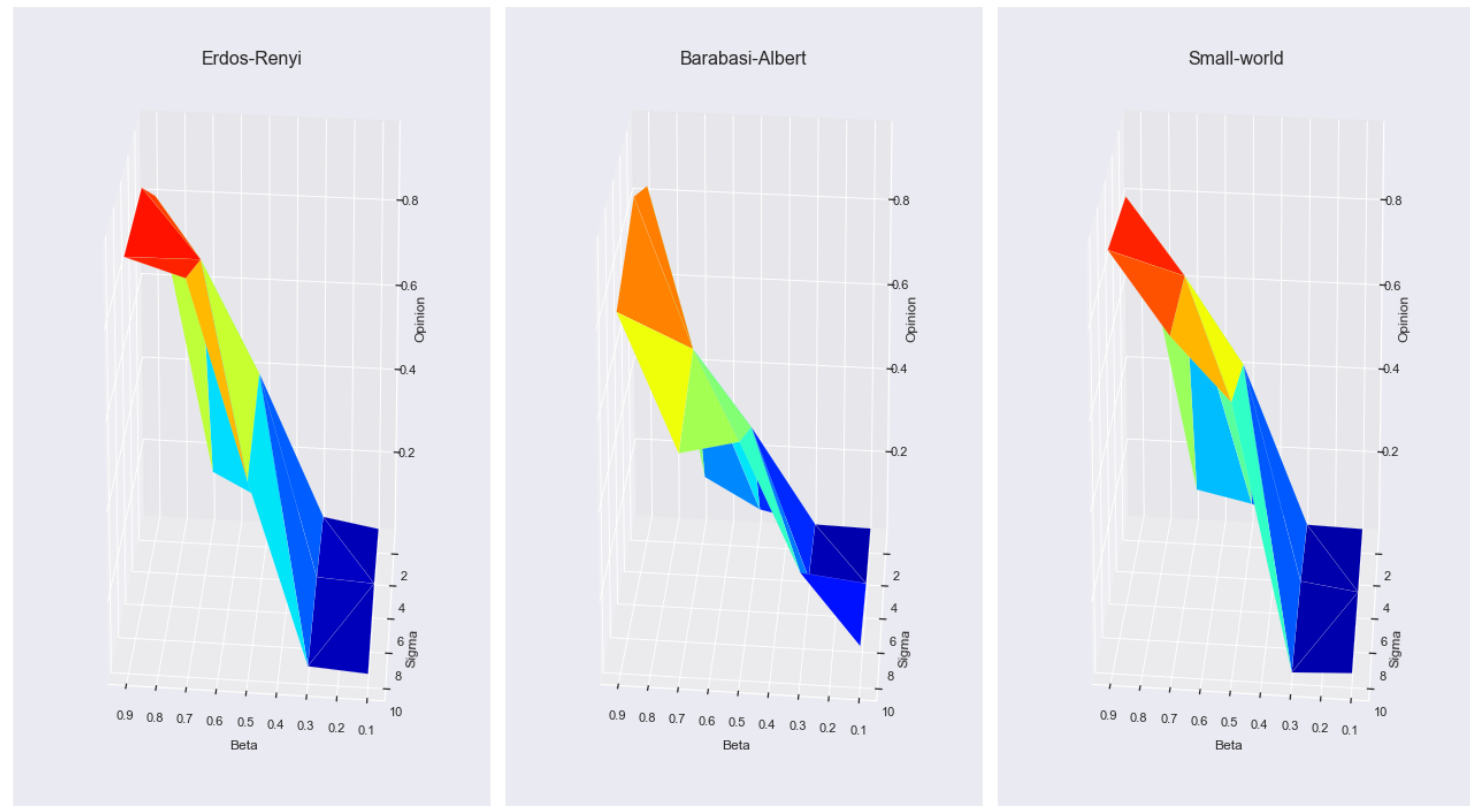

Figura 1. Overall average opinion for different values for $\sigma$ (sigma) and $\beta$ (beta) in a scenario with no interventions. Red surfaces show higher values for the opinions, while blue surfaces are lower values for opinions in each of the 3 studied topologies (Erdos-Renyi, Barabási-Albert and Small-world)

Figure 2 shows the average and standard deviation of the 100 runs for each network topology without interventions. It is apparent that the turning point from negative opinions on social distancing adherence to positive opinions is $\beta>0.5$ and $\sigma>5$, being the only exception the case when $\beta=0.7$ and $\sigma=1$. That would be explained by the fact that such a low $\sigma$ would slow down the received influence from their peers, and even though $\beta$ is high, it would not be sufficient to provide a positive outcome. The graphics in Figure 2 also show that the closer the parameters are to the extremes ( 0 or 1$)$, the lower the standard deviation of the results.

The simulations also aim to understand how can the spread of positive opinions about the social distancing be improved. For that we used a rate of $10 \%$ of the people in the network to become targets of the intervention. For this $10 \%$ the opinion is fixed to 1 , meaning that these people are always partaking the social distancing. Table 1 shows in bold the strategies that worked the best for each combination of $(\beta, \sigma)$ in each network topology. For the Barabasi-Albert topology, using the betweenness centrality as a target criteria has shown to boast the overall opinion in almost all scenarios. The eigenvector centrality, on the other hand, it is the criteria providing better results for a Erdos-Renyi topology. For the Small-world topology, betweenness centrality is a good choice if $\beta<$ 0.5 , and eigenvector centrality otherwise. Closeness centrality was the best option in only 2 out of the 81 possibilities of intervention.

The codes, results obtained and other analysis discussed in this arti- 

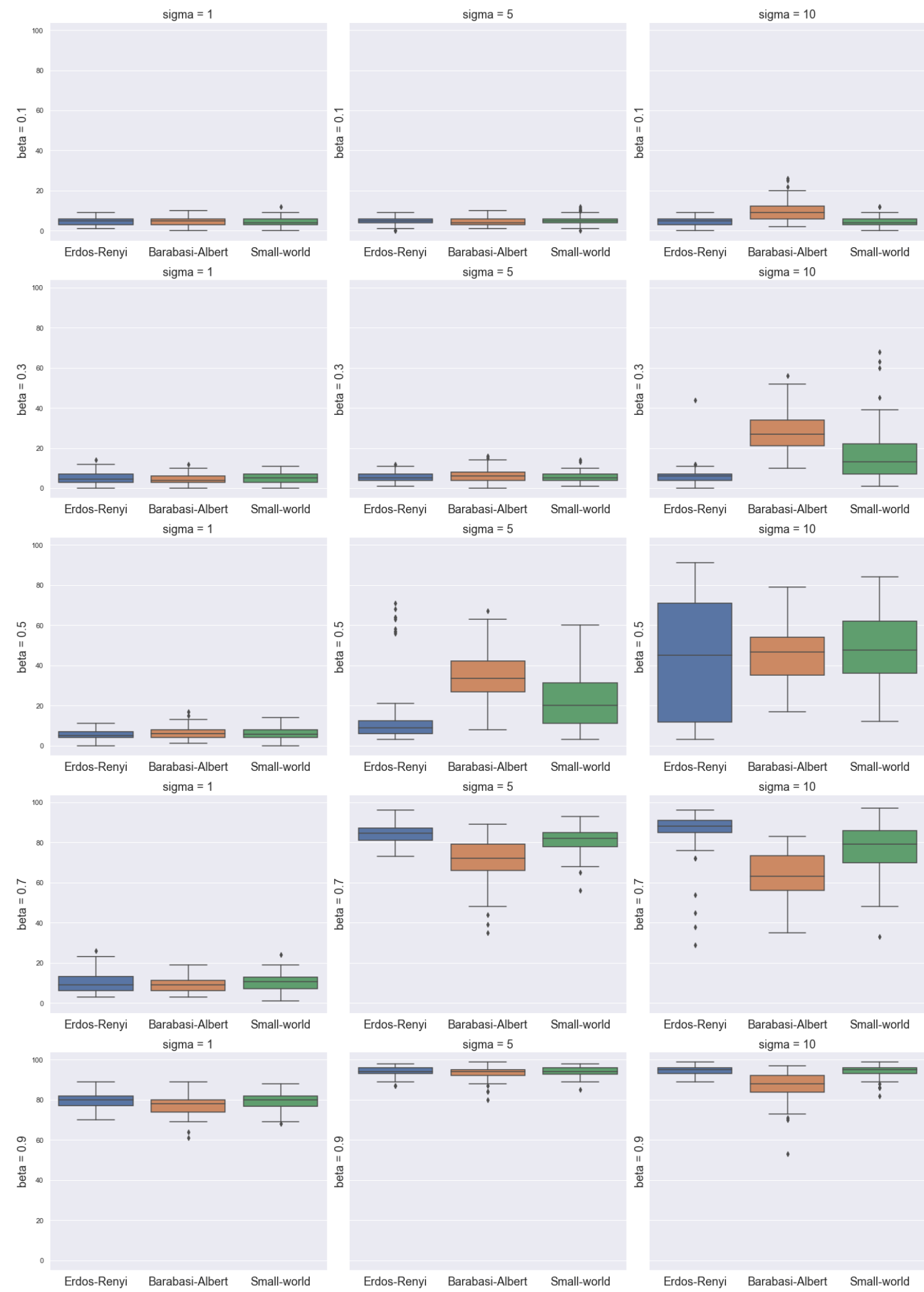

Figura 2. Percentage of the agents in social distancing by the end of the simulation with no interventions for different combinations of $\beta$ and $\sigma$. 


\begin{tabular}{|c|c|c|c|c|c|c|c|c|c|c|}
\hline \multicolumn{2}{|c|}{ Network } & \multicolumn{3}{|c|}{ Barabasi-Albert } & \multicolumn{3}{|c|}{ Erdos-Renyi } & \multicolumn{3}{|c|}{ Small-world } \\
\hline Beta & Sigma & Betweenness & Closeness & Eigenvector & Betweenness & Closeness & Eigenvector & Betweenness & Closeness & Eigenvector \\
\hline 0.1 & 1 & $334.68 \%$ & $324.01 \%$ & $330.05 \%$ & $301.79 \%$ & $302.91 \%$ & $300.30 \%$ & $314.44 \%$ & $312.72 \%$ & $313.16 \%$ \\
\hline 0.1 & 5 & $699.24 \%$ & $620.07 \%$ & $614.18 \%$ & 323.53\% & $320.75 \%$ & $320.05 \%$ & $344.49 \%$ & $340.61 \%$ & $343.90 \%$ \\
\hline 0.1 & 10 & $527.19 \%$ & $468.44 \%$ & $452.33 \%$ & $336.43 \%$ & $336.90 \%$ & $350.78 \%$ & $436.68 \%$ & $411.22 \%$ & $408.19 \%$ \\
\hline 0.5 & 1 & $543.81 \%$ & $486.29 \%$ & $467.28 \%$ & $319.27 \%$ & $319.67 \%$ & $321.96 \%$ & $322.15 \%$ & $310.88 \%$ & $305.45 \%$ \\
\hline 0.5 & 5 & $204.32 \%$ & $186.58 \%$ & $180.12 \%$ & $437.25 \%$ & $419.74 \%$ & $443.35 \%$ & $244.12 \%$ & $234.38 \%$ & $225.52 \%$ \\
\hline 0.5 & 10 & $165.22 \%$ & $157.12 \%$ & $155.05 \%$ & $161.52 \%$ & $161.52 \%$ & $162.56 \%$ & $139.61 \%$ & $138.12 \%$ & $135.36 \%$ \\
\hline 0.9 & 1 & $106.26 \%$ & $105.31 \%$ & $104.73 \%$ & $103.70 \%$ & $103.59 \%$ & $103.66 \%$ & $103.21 \%$ & $103.14 \%$ & $103.53 \%$ \\
\hline 0.9 & 5 & $100.55 \%$ & $100.54 \%$ & $100.92 \%$ & $100.56 \%$ & $100.79 \%$ & $100.58 \%$ & $100.66 \%$ & $100.67 \%$ & $100.75 \%$ \\
\hline 0.9 & 10 & $106.12 \%$ & $105.15 \%$ & $105.45 \%$ & $100.39 \%$ & $100.41 \%$ & $100.50 \%$ & $101.11 \%$ & $100.55 \%$ & $100.69 \%$ \\
\hline
\end{tabular}

Tabela 1. Improvement of the overall opinion about adhering to social distancing after applying network-oriented interventions (betweenness, closeness and eigenvector centralities) in different network topologies (Barabási-Albert, Erdos-Renyi and Small-world).

cle can be found in the repository https://github.com/ericinlinux/ covid-social-contagion.

\section{Discussion}

We presented an network-oriented model for the spread of opinions about social distancing behaviour adherence in a situation of a pandemic. The model used differential equations to describe how opinions spread based on peer interactions of two or more people in a network. This study is theoretical but inspired by the current situation of the world caused by the COVID-19 spread. This is an initial work investigating how the spread of opinions will interfere on the contamination rates using social networks as the representation of how people interact with each other.

Regarding the Question 1, in Section 1, the results shown in here are two-folded. There is little evidence to say that one of the three network topologies favour the spread of the opinions better, but presents as an important outcome the necessity of engagement of the health authorities if they want to increase faster the adherence to social distancing of the general population. That is observed by the influence of $\beta$ on the changes from a negative community opinion to a positive stand.

This work also have shown how interventions based on network measures could increase the overall opinion of the whole group (Question 2, in Section 1). By targeting $10 \%$ of the population and adapting their social distancing behaviour improves the overall opinion from $100 \%$ up to $700 \%$. The costs of the interventions will be considered in future work, as well as different percentages of targeted people as it is important to define a better trade-off for governmental policies with optimal outcomes.

The model for the spread of the virus [Klôh et al. 2020] is also going to be incorporated to the model in future investigations, so we can understand how the model on opinions will affect the infection rates based when part of the population partaking in the protocol or not. Other aspects that will be included in the model are the obligatory social distancing in situations where people are penalised in case they break the protocol and how it would affect the whole society.

\section{Referências}

Araújo, E. (2018). Contagious: Modeling the Spread of Behaviours, Perceptions and Emotions in Social Networks. PhD thesis, Vrije Universiteit Amsterdam. 
Araújo, E. and Treur, J. (2016). Analysis and refinement of a temporal-causal network model for absorption of emotions. In International Conference on Computational Collective Intelligence, pages 27-39. Springer.

Barabási, A.-L. and Albert, R. (1999). Emergence of scaling in random networks. science, 286(5439):509-512.

Bosse, T., Duell, R., Memon, Z. A., Treur, J., and Van Der Wal, C. N. (2009). A multiagent model for emotion contagion spirals integrated within a supporting ambient agent model. In International Conference on Principles and Practice of Multi-Agent Systems, pages 48-67. Springer.

Braunack-Mayer, A., Tooher, R., Collins, J. E., Street, J. M., and Marshall, H. (2013). Understanding the school community's response to school closures during the h1n1 2009 influenza pandemic. BMC public health, 13(1):344.

Centola, D. (2018). How behavior spreads: The science of complex contagions, volume 3. Princeton University Press.

Chhatwal, J. and He, T. (2015). Economic evaluations with agent-based modelling: an introduction. Pharmacoeconomics, 33(5):423-433.

Dandekar, R. and Barbastathis, G. (2020). Quantifying the effect of quarantine control in covid-19 infectious spread using machine learning. medRxiv.

Erdős, P. and Rényi, A. (1960). On the evolution of random graphs. Publ. Math. Inst. Hung. Acad. Sci, 5(1):17-60.

Fang, Y., Nie, Y., and Penny, M. (2020). Transmission dynamics of the covid-19 outbreak and effectiveness of government interventions: A data-driven analysis. Journal of Medical Virology, 92(6):645-659.

Freeman, L. C. (1989). Social networks and the structure experiment. Research methods in social network analysis, pages 11-40.

Hennig, M., Brandes, U., Pfeffer, J., and Mergel, I. (2012). Studying social networks: A guide to empirical research. Campus Verlag.

Klôh, V., Silva, G., Ferro, M., Araújo, E., de Melo, C. B., de Andrade Lima, J. R. P., and Martins, E. R. (2020). The virus and socioeconomic inequality: An agent-based model to simulate and assess the impact of interventions to reduce the spread of covid-19 in rio de janeiro, brazil. Brazilian Journal of Health Review (BJRH), pages $3647-3673$. http://www.brjd.com.br/index.php/BJHR/article/view/9209.

Koo, J. R., Cook, A. R., Park, M., Sun, Y., Sun, H., Lim, J. T., Tam, C., and Dickens, B. L. (2020). Interventions to mitigate early spread of sars-cov-2 in singapore: a modelling study. The Lancet Infectious Diseases.

Kucharski, A., Russell, T., Diamond, C., Liu, Y., Edmunds, J., Funk, S., Eggo, R., Sun, F., Jit, M., Munday, J., Davies, N., Gimma, A., Zandvoort, K., Gibbs, H., Hellewell, J., Jarvis, C., Clifford, S., Quilty, B., Bosse, N., and Flasche, S. (2020). Early dynamics of transmission and control of covid-19: a mathematical modelling study. The Lancet Infectious Diseases. 
Li, L., Yang, Z., Dang, Z., Meng, C., Huang, J., Meng, H., Wang, D., Chen, G., Zhang, J., Peng, H., and Shao, Y. (2020). Propagation analysis and prediction of the covid-19. Infectious Disease Modelling, 5:282 - 292.

Lin, Q., Zhao, S., Gao, D., Lou, Y., Yang, S., Musa, S. S., Wang, M. H., Cai, Y., Wang, W., Yang, L., and He, D. (2020). A conceptual model for the coronavirus disease 2019 (covid-19) outbreak in wuhan, china with individual reaction and governmental action. International Journal of Infectious Diseases, 93:211 - 216.

McPherson, M., Smith-Lovin, L., and Cook, J. M. (2001). Birds of a feather: Homophily in social networks. Annual review of sociology, 27(1):415-444.

Saunders-Hastings, P., Hayes, B. Q., et al. (2017). Modelling community-control strategies to protect hospital resources during an influenza pandemic in ottawa, canada. PloS one, 12(6).

Sokolowski, J. A. and Banks, C. M. (2011). Principles of modeling and simulation: a multidisciplinary approach. John Wiley \& Sons.

Treur, J. (2020). NETWORK-ORIENTED MODELING FOR ADAPTIVE NETWORKS: Designing Higher-order Adaptive Biological,... Mental and Social Network Models. Springer.

van Woudenberg, T., Simoski, B., Araújo, E., Bevelander, K., Burk, W., Smit, C., Buijs, L., Klein, M., and Buijzen, M. (2019). Simulated social network interventions to promote physical ativity: Who should be the influence agents? Journal of Medical Internet Research, 21.

Walker, P. G., Whittaker, C., Watson, O., Baguelin, M., Ainslie, K., Bhatia, S., Bhatt, S., Boonyasiri, A., Boyd, O., Cattarino, L., et al. (2020). The global impact of covid-19 and strategies for mitigation and suppression. Technical Report 12, Imperial College London.

Watts, D. J. and Strogatz, S. H. (1998). Collective dynamics of 'small-world' networks. nature, 393(6684):440-442.

Webster, R. K., Brooks, S. K., Smith, L. E., Woodland, L., Wessely, S., and Rubin, G. J. (2020). How to improve adherence with quarantine: Rapid review of the evidence. Public Health.

Wolfram, C. (2020). An agent-based model of covid-19. Complex Systems, 29:87-105.

Zhou, X., Ma, X., Hong, N., Su, L., Ma, Y., He, J., Jiang, H., Liu, C., Shan, G., Zhu, W., Zhang, S., and Long, Y. (2020). Forecasting the worldwide spread of covid-19 based on logistic model and seir model. medRxiv. 